\title{
Diaphragmatic Plication in a Mechanically Ventilated Patient with Phrenic Nerve Paralysis and Severe Pre-Operative Respiratory Insufficiency
}

\author{
Lucia Morelli $^{\text {a }}$, Enrico Ferrari ${ }^{\text {a }}$, Federico Mazza ${ }^{\text {b }}$, Massimiliano Venturino ${ }^{\text {b }}$, Mattia \\ Manitto $^{\text {a }}$, Giovanni B. Ratto ${ }^{\text {a }}$ \\ ${ }^{a}$ Department of Thoracic Surgery, University San Martino Hospital and National Cancer Research \\ Institute, Largo Rosanna Benzi 10, Genova, Italy \\ ${ }^{\mathrm{b}}$ Department of Thoracic Surgery, Santa Corona Hospital, Pietra Ligure, Savona, Italy
}

\begin{abstract}
:
Introduction: we propose the objective revision of a critical clinical case which presented so important lifethreatening complications.
\end{abstract}

Presentation of Case: we present a case of patient underwent cardiovascular surgery, developing diaphragmatic paralysis and dependence on mechanical ventilation.

Discussion: The patient was weaned from mechanical ventilation after diaphragmatic plication, in spite of severe pre-operative respiratory insufficiency.

Conclusion: This case let us re-valuate the surgical indication of diaphragmatic plication for weaning a patient from a mechanical ventilation.

Keywords: phrenic nerve paralysis, diaphragmatic plication, mechanical ventilation

\section{INTRODUCTION}

Diaphragmatic plication (DP) for phrenic nerve palsy occurring after cardiac surgery is infrequently performed in adults, despite its successful application in children [1]. Paucity of the relevant literature is one of the main barriers to the widespread adoption of DP in adult patients. In this setting, only few reports deal with DP to wean from mechanical ventilation in patients with severe respiratory insufficiency [1]. We present a case of failure to wean from mechanical ventilation, successfully treated by DP in a patient with postcardiotomy phrenic nerve paralysis and severe respiratory insufficiency.

\section{MATERIALS AND METHODS}

A 70-year-old man was admitted with diagnosis of distal aortic arch aneurysm $(8 \times 10 \times 8 \mathrm{~cm})$ extending from the origin of left subclavian artery to the descending aorta. Cardiac ejection fraction was $60 \%$, forced vital capacity $66 \%$, forced expiratory volume in one second $50 \%, \mathrm{PaO} 252 \mathrm{mmHg}$ and $\mathrm{PaCO} 2$ $43 \mathrm{mmHg}$, respectively.

Through a median sternotomy and left anterior thoracotomy a cardiopulmonary by-pass was instituted. A neurysmectomy, replacement of the aortic arch and proximal descending aorta $(30 \mathrm{~mm}$ vascular graft: Intervascular; WL Gore \& Associates Inc, USA) and button re implantation of the left subclavian, left common carotid and right brachiocephalic artery were carried out. Extubated on the second post-operative day (pod), the patient showed signs of left hemiplegia with brain Computed Tomography (CT) evidence of right parietal and frontal lesions.

Re-intubated for a severe respiratory distress syndrome requiring mechanical ventilation, the patient showed diffuse left lung atelectasis and elevation of the left hemi-diaphragm (Figures 1a, 2a). Cardiac ultrasound demonstrated preserved cardiac function with ejection fraction of $58 \%$. Despite tracheostomy, several attempts of mechanical ventilation weaning were unsuccessful. On the eight 
pod the patient underwent DP. Through a muscle-sparing thoracotomy, a row of longitudinal, twozero polypropylene $\mathrm{U}$ sutures placed in a posterior to anterior direction, reinforced at both ends with pledgets was performed. To make the diaphragm taut and flat, a second plication was added. To allow the complete pulmonary re-expansion, lysis of adhesions was done. Immediate chest X-ray showed the correct position of the left diaphragm. On the first pod, signs of hypovolemic shock developed. CT scan showed left pleural effusion, with lung atelectasis, in spite of two $28 \mathrm{Ch}$ chest drains. Blood clots were removed through a re-sternotomy; no site of active bleeding was found. On the fourth pod, signs of peritonitis and hypovolemic shock developed. CT scan showed a large peri- end intra splenic hematoma. Splenectomy was performed; no correlation between diaphragmatic plication and splenic bleeding was evident. Extubated on the third pod the patient progressively recovered. Six months after plication, a chest CT demonstrated the good expansion of left lung and the correct position of the diaphragm (Figures 1b, 2b). Forced vital capacity and forced expiratory volume in one second were $55 \%$ and $45 \%$, respectively.
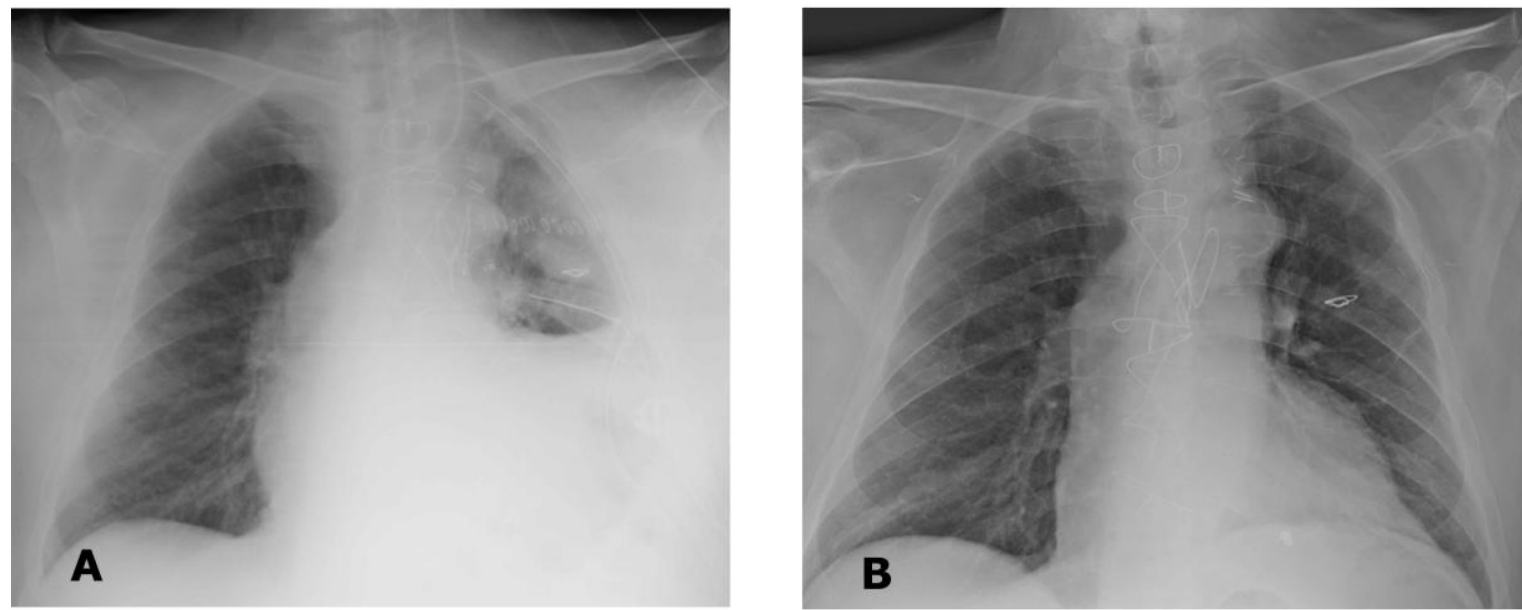

Figure1. Chest X-Ray demonstrates the elevation of left paralysed hemidiaphragm after cardiac surgery, with consensual lung atelectasis (a) and the correct position of left hemidiaphragm after Diaphragmatic Plication, with good lung expansion (b).
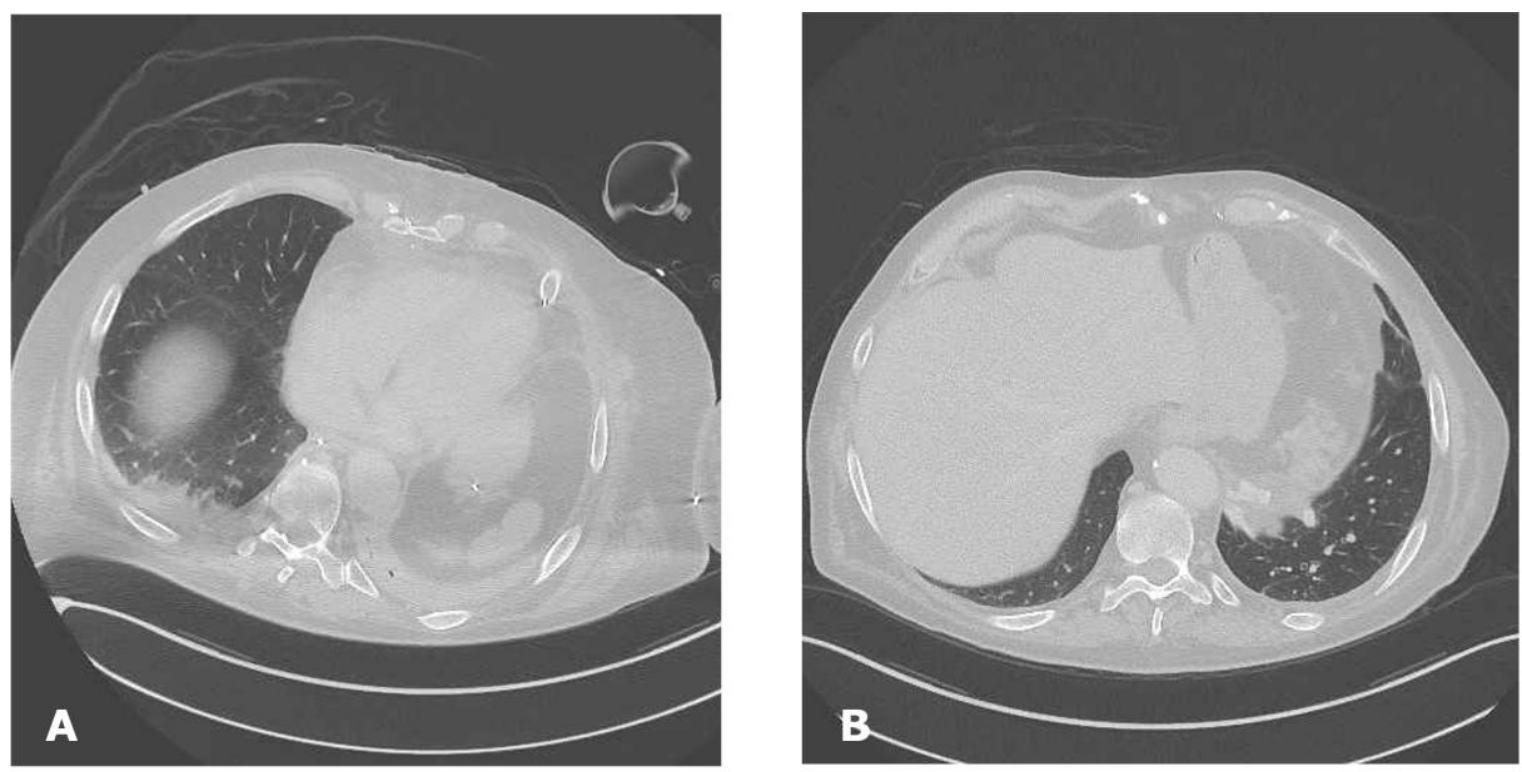

Figure2. Thoracic CT shows the elevation of left paralysed hemidiaphragm after cardiac surgery, with splenic migration in thorax $(\boldsymbol{a})$ and the correct position of left hemidiaphragm in comparison with right diaphragm, after Diaphragmatic Plication, without the migration of splanchnic organs and with good lung expansion (b).

\section{RESUlTS AND DisCuSSION}

Immediate diaphragmatic plication was not performed, since it has been reported that adult patients are generally able to compensate and mechanical ventilation is rarely required [1]. Whether immediate DP could have prevented respiratory distress syndrome from developing may not be inferred from the present experience. In our patient, once re-intubation and mechanical ventilation 
were required, the benefits and risks of DP were uncertain, due to the association of severe preoperative respiratory insufficiency and several contributing factors other than diaphragmatic palsy (pneumonia, atelectasis, and left hemiplegia). Although the clinical presentation and surgical treatment (DP) performed in our patient were fairly standard, the present report suggests that DP should be attempted even in patients with severe pre-operative respiratory insufficiency. Weaning from mechanical ventilation was achieved in spite of several factors potentially worsening prognosis, and the development of post-operative complications.

A huge number of technical variations have been described for DP. Although the technique we adopted for DP achieved the purpose of mechanical ventilation weaning, two major complications occurred. The haemothorax was likely due to adhesion lysis and anticoagulant drug administration. Even if the cause of splenic bleeding was not evident at laparotomy, the spleen injury was possibly related to diaphragmatic suturing. To avoid splenic injury, an initial $5 \mathrm{~cm}$ diaphragm transection was suggested [2]. A case of an obese woman with splenic bleeding after PD was reported [3]. Our experience shows that splenic bleeding may occur in spite of the fact that large sutures have not been used, the patient was not obese, and the diaphragm had normal thickness.

Good results have been reported with minimally invasive diaphragmatic plication (VATS plication) [4]. Our experience suggests that a muscle sparing thoracotomy provides a wide workspace, shortens surgery duration, and allows obtaining the desired diaphragmatic tension.

Many studies report that after DP, in non-ventilated patients, spirometric and static lung volumes values return to normal range [6,7]. Welvaart et al. performed cardiopulmonary exercise tests before and after DP, in non-ventilated patients with mainly post-operative paralysis of diaphragm. They concluded FEV1 (62\% pre-operative versus $72 \%$ post-operative of predictive value) and FVC (67\% vs $77 \%$ of predictive value) significantly increased and the ventilatory frequency decreased, but maximal exercise capacity did not change. In non-ventilated adult patients with progressive dyspnoea DP could obtain excellent results [8].

Only few reports deal with DP in mechanically ventilated adult patients, making the benefit of DP unclear and not standardized [4, 9]. In spite of isolated case reports supporting DP to wean from mechanical ventilation [4], Simansky et al reported that only one out of four plicated mechanically ventilated adult patients was weaned [1]. The authors concluded that DP offers limited benefit in weaning ventilated adults and it is probably not indicated in patients with diaphragmatic paralysis and severe pulmonary parenchymal disease. In the Kuniyoshi et al. series, 4 patients underwent DP to wean from mechanical ventilation [5]. One of these patients suffered from chronic obstructive pulmonary disease (VC: 75\%; FEV1: 56\%; $\mathrm{PaO} 2: 51 \mathrm{~mm} \mathrm{Hg}$ ) and died 15 days after plication.

\section{Conclusions}

At variance with the reported literature, our experience suggests that DP has the potential for improving ventilation and allowing weaning from respiratory support even in patients with severe preoperative respiratory insufficiency. The present report should hopefully encourage surgeons to a more widespread use of this therapeutic option even in complex situations.

\section{REFERENCES}

[1] Simansky DA, Paley M Refealy Y, Yellin A. Diaphragm plication following phrenic nerve injury: a comparison of paediatric and adult patients. Thorax 2002; 57: 613-6.

[2] Celik S, Celik M, Aydemir B, Tunckaya C, Okay T et al Long-term results of diaphragmatic plication in adults with unilateral diaphragm paralysis. J Cardiothorac Surg 2010; 5: 111-18.

[3] Pathak $S$ et Page RD. Splenic injury followig diaphragmatic plication: an avoidable lifethreatening complication. Interact Cardiovasc Thorac Surg 2009; 9: 1045-6.

[4] Tsakiridis K, Visuoli AN, Zarogoulidis P, Machairiotis N, Christofis C et al. Early hemidiaphragmatic plication trough a video assisted minithoracotmy in postcardiotomy phrenic nerve paresis. J Thorac Dis 2012; 4 (SI) 56-68.

[5] Kuniyoshi Y, Yamashiro S, Miyagi K, Uezu T, Arakaki K et al. Diaphragmatic plication in adult patients with diaphragm paralysis after cardiac surgery. Ann Thorac Cardiovasc Surg 2004; 10: $160-6$. 
[6] Freeman RK, Van Voerkom J, Vyverberg A, Ascioti A. Long term follow up of the functional and physiologic results of diaphragmatic plication in adults with unilateral diaphragmatic paralysis. Ann Thorac Surg 2009; 88: 1112-7.

[7] Higgs SM, Hussain A, Jackson M, Donnelly RJ, Berrisford RG. Long term results of diaphragmatic plication for unilateral diaphragm paralysis. Eur J Cardiothorac Surg 2002; 21: 294-7.

[8] Welvaart WN, Jak PMC, van de Veerdonk MC, Marcus JT, Ottenheijm CAC et al. Effects of diaphragm plication on pulmonary function and cardiopulmonary exercise parameters. Eur $\mathbf{J}$ Cardiothorac Surg 2013; 44: 643-7.

[9] Takeda S, Nakahara k, Fujii Y, Minami M, Matsuda H. Plication of paralyzed hemidiaphragm after right sleeve pneumonectomy. Ann Thorac Surg 1994; 58 (6): 1755-7; discussion 1757-8. 